\title{
DESENVOLVIMENTO REGIONAL E RESILIÊNCIA DE CLUSTER: UMA PROPOSTA TEÓRICA
}

REGIONAL DEVELOPMENT AND CLUSTER RESILIENCE: A THEORETICAL PROPOSAL

Recebido em 23.02.2019. Aprovado em 27.02.2019

Avaliado pelo sistema double blind review

DOI: http://dx.doi.org/10.12712/rpca.v13i1.28142

\section{Vitor Klein Schmidt}

Vitorks1993@hotmail.com

Programa de Pós-Graduação em Administração/Universidade Federal do Rio Grande do Sul (UFRGS), Porto Alegre/RS, BRASIL

ORCID: https://orcid.org/0000-0002-8493-4331

\section{Aurora Carneiro Zen}

Aurora.zen@ufrgs.br

Programa de Pós-Graduação em Administração/Universidade Federal do Rio Grande do Sul (UFRGS), Porto Alegre/RS, BRASIL

ORCID: orcid.org/0000-0003-4078-4359

\section{Resumo}

Clusters têm sido objeto de pesquisa durante décadas devido as vantagens competitivas locais que impulsionam o desenvolvimento regional. Entretanto, grande parte dos estudos desconsideraram o desenvolvimento de suas estruturas ao longo do tempo. Recentemente, acadêmicos passaram a desenvolver abordagens dinâmicas que conseguissem explicar os padrões de desenvolvimento dos clusters. A resiliência de cluster é um constructo utilizado para entender como os cluster adaptam as suas estruturas a fim de superar choques, entretanto ainda é um conceito bastante embrionário. O objetivo deste artigo é analisar a resiliência econômica dos clusters, buscando identificar os principais elementos que impactam na sua capacidade de resistência, recuperação e adaptação após um choque externo. Para tanto, foi desenvolvido um framework teórico baseado nos recentes avanços da literatura de geografia econômica evolucionária. Como principais contribuições, este artigo redefiniu resiliência de cluster e seus principais elementos.

Palavras-chave: Cluster. Resiliência. Desenvolvimento regional. Choque.

\begin{abstract}
Clusters have been the subject of research for decades because of the local competitive advantages that promote regional development. However, most studies disregard the development of their structures over time. Recently, academics began to develop dynamic approaches that could explain the patterns of development of the clusters. Cluster resilience is a construct used to understand how clusters adapt their structures in order to overcome shocks, but it is still a rather embryonic concept. The objective of this article is to analyze the economic resilience of clusters, seeking to identify the main elements that impact on their resilience, recovery and adaptation after an external shock. For that, a theoretical framework was developed based on recent advances in the literature of evolutionary economic geography. As key contributions, this article still redefined cluster resilience and its main elements.
\end{abstract}

Keywords: Cluster. Resilience. Regional development. Shock. 


\section{Introdução}

As aglomerações geográficas de firmas têm recebido crescente atenção nas últimas décadas, sendo denominadas principalmente como clusters. A existência de um cluster facilita a difusão de conhecimentos, oferece mão de obra qualificada e fornecedores especializados (MOROSINI, 2004). Desta forma, os clusters são uma estrutura econômica importante, uma vez que auxiliam na promoção do desenvolvimento econômico regional, através de ganhos coletivos que, dificilmente, as firmas teriam acesso agindo de forma isolada (SCHMITZ, 1999). Apesar do reconhecimento da importância dos clusters, a maior parte dos estudos adotaram uma visão estática, desconsiderando o desenvolvimento desses arranjos ao longo do tempo (Ter WAL; BOSCHMA, 2011), tratando-os como estruturas econômicas que levariam ao sucesso regional (MARTIN; SUNLEY, 2006). Nos últimos dez anos, diversos pesquisadores passaram a adotar uma abordagem dinâmica, levando em consideração as formas como os clusters se originam, desenvolvem, declinam e se renovam (MENZEL; FORNAHL, 2010; MARTIN; SUNLEY, 2011). Inspirada pelos avanços da teoria evolucionária (BOSCHMA; FRENKEN, 2006), as novas abordagens consideram os cluster como estruturas dinâmicas que se transformam ao longo do tempo.

A abordagem baseada na resiliência econômica possui a sua origem na geografia econômica evolucionária e visa explicar o processo de transformação, bem como o porquê de algumas regiões conseguirem se recuperar rapidamente de choques econômicos, enquanto outras perecem (MARTIN, 2012; MARTIN; SUNLEY, 2015; BOSCHMA, 2015). Sistemas econômicos são confrontados constantemente por choques que irão impactar na sua trajetória de desenvolvimento (MARTIN; SUNLEY, 2015). A evolução e o sucesso de um sistema econômico serão influenciados tanto pelas suas habilidades de criar elementos que suportem o seu crescimento, como também as suas capacidades de responder positivamente aos choques (HOLM; ØSTERGAARD, 2015). O conceito de resiliência não foca apenas no sucesso econômico, mas sim em mantê-lo, através de um longo período de tempo, adaptando o sistema às necessidades que cada distúrbio exige (ERAYDIN, 2016).

O objetivo deste artigo é analisar a resiliência econômica dos clusters, buscando identificar os principais elementos que impactam na sua capacidade de resistência, recuperação e adaptação após um choque externo. Para tanto, este ensaio teórico também apresenta um framework com os diversos elementos importantes para o desenvolvimento e sustentação de um cluster. Apesar do crescente interesse na dinâmica dos clusters ao longo do seu ciclo de vida, ainda se observa uma lacuna na literatura sobre os elementos da resiliência de clusters. Este trabalho torna-se relevante, pois a compreensão dos elementos que impulsionam ou limitam o desenvolvimento de clusters frente a choques pode auxiliar políticas públicas futuras que visam o desenvolvimento de clusters. Tal análise torna-se ainda mais pertinente para o contexto brasileiro, diante da grave crise econômica enfrentada pelo país nos últimos anos (OREIRO, 2017).

\section{Referencial Teórico}

\section{Declínio e renovação de clusters}

A literatura sobre clusters destaca que as características locais contribuem para o aumento do desempenho regional, através da criação de externalidades as quais são acessadas unicamente pelas firmas localizadas dentro da região do cluster. Desta forma, as firmas conseguem alcançar maiores níveis competitivos devido ao ganho de eficiência que advém da ação coletiva (SCHMITZ, 1999). As principais externalidades observadas são as altas concentrações de mão de obra especializada, o desenvolvimento local de infraestrutura, presença de fornecedores especializados e a melhora do fluxo de informações (MOROSINI, 2004). Porter (1998) apresenta a definição de cluster mais difundida dentro da literatura. Segundo o autor, clusters são: concentrações geográficas de companhias interconectadas e instituições 
de um campo particular. Apesar de relevante, a definição de Porter (1998) foi duramente criticada por ser vaga e ambígua (MARTIN; SUNLEY, 2011).

Desde os anos 2000, a Geografia Econômica Evolucionária (GEE) vem recebendo muito interesse dos pesquisadores da área de aglomerações geográficas de firmas (BOSCHMA, 2005; BOSCHMA; MARTIN, 2010; MARTIN; SUNLEY, 2006). A GEE auxilia a explicar como uma região se modifica, demonstrando como a geografia importa ao determinar a natureza e a trajetória da evolução dos sistemas econômicos, enfatizando os mecanismos que facilitam ou limitam a adaptação econômica de um território (BOSCHMA; FRENKEN, 2006). Os estudos sobre clusters foram fortemente influenciados pela GEE, pesquisadores passaram a discutir o caráter evolucionário de desenvolvimento dos clusters, inicialmente através da metáfora de ciclo de vida (MENZEL; FORNAHL, 2010) e, mais recentemente, resiliência de cluster (WROBEL, 2013).

A abordagem de ciclo de vida de cluster enfatiza o desenvolvimento dos clusters ao longo do tempo, explicando como os clusters emergem, se desenvolvem, declinam e se renovam (MENZEL; FORNAHL, 2010). Já a resiliência de cluster foca, principalmente, no processo de declínio e renovação desses arranjos. O declínio dos clusters têm sido tradicionalmente explicados através do efeito de lock-in negativo (MARTIN; SUNLEY, 2006). O efeito lock-in negativo se refere ao aprisionamento político, funcional e cognitivo em uma dada trajetória de desenvolvimento (GRABHER, 1993). O efeito lock-in ocorre em função da dificuldade de uma economia regional em romper com o seu legado histórico e a sua tendência em reproduzir as mesmas estruturas e tecnologias ao longo do tempo. Tal dificuldade se acentua ainda mais devido à geração de retornos crescentes e de externalidades que influenciaram historicamente o processo de desenvolvimento dos clusters (MARTIN; SUNLEY, 2006).

Apesar do forte argumento de que os clusters declinam devido à inércia promovida pelo efeito lock-in, o conceito em si não se refere somente ao declínio, mas também à emergência e ao desenvolvimento de economias regionais (MARTIN; SUNLEY, 2006), bem como o processo de adaptação e transformação dos clusters (BOSCHMA; MARTIN, 2010; TREADO, 2010; BOSCHMA, 2015). Ao estudar o processo de declínio e renovação dos clusters, Hassink (2010) e Yang et al. (2017) destacam a forma pela qual as networks se aprisionam, subdividindo o efeito lock-in em lock-in forte e lock-in fraco. O lock-in forte se refere ao aprisionamento descrito por Grabher (1993), no qual o forte enraizamento local impede o cluster de se renovar. Já o lock-in fraco se refere à fraca resistência local para a reestruturação econômica. As relações existentes dentro de um cluster com um lock-in fraco não possuem a tendência de preservar as estruturas econômicas existentes (HASSINK, 2010).

Dentro da perspectiva evolucionária, a renovação dos clusters está relacionada diretamente com a capacidade do cluster em introduzir novos conhecimentos, a fim de romper com a trajetória dominante e assumir novas rotinas (TÖDTLING; TRIPPL, 2005; TRIPPL; OTTO, 2009; MENZEL; FORNAHL, 2010). Nessa perspectiva, novos recursos são integrados ao processo produtivo do cluster e recombinados aos recursos já existentes, oferecendo novas oportunidades de exploração econômica ao cluster (MARTIN; SUNLEY, 2006). A renovação do cluster abre espaço para a introdução de inovações (MARTIN; SUNLEY, 2011), novas empresas (TER WAL; BOSCHMA, 2011), ou ainda, novos setores, podendo eles estar ou não relacionados com o cluster (FRENKEN et al., 2007).

Existem diversos modelos que apresentam as formas pelas quais os clusters se renovam (TRIPPL; OTTO, 2009; MENZEL; FORNAHL, 2010; MARTIN; SUNLEY, 2011). Cada modelo é dividido em tipologias diferentes, mas mantêm uma hierarquia que relaciona o processo de transformação do cluster e a introdução de novos conhecimentos. Trippl e Otto (2009) apresentam um dos modelos mais coerentes com a GEE sobre renovação de cluster. Dentro do modelo, um cluster pode se renovar de três maneiras distintas. A primeira maneira, denominada "Mudança Incremental", está relacionada com uma pequena mudança na sua trajetória. Essa mudança ocorre por meio da inserção de uma pequena quantidade de novos conhecimentos, os quais permitem com que o cluster melhore o seu desempenho dentro do seu atual mercado (TRIPPL; OTTO, 2009). Essa primeira maneira de renovação consiste, 
principalmente, em uma reciclagem criativa, adaptando velhos conhecimentos em novas práticas (TRIPPL; OTTO, 2009).

A segunda maneira de renovação se chama "Diversificação" e envolve uma maior quantidade de mudanças do que uma mera regeneração de velhas rotinas (TRIPPL; OTTO, 2009). A diversificação está relacionada a um aumento da heterogeneidade local (MENZEL; FORNAHL, 2010), através da emergência de novos setores ou clusters na região. Estas novas firmas, por sua vez, devem ser capazes de se direcionarem para novos setores, recombinando capacidades e características locais (TRIPPL; OTTO, 2009). Por fim, a terceira maneira de renovação, "Mudança Radical", envolve um grande aumento nas bases tecnológicas dos cluster (MENZEL; FORNAHL, 2010), normalmente envolvendo a atração e a clusterização de setores extensivos em conhecimentos (TRIPPL; OTTO, 2009). A introdução de novos conhecimentos e a mudança setorial faz com que o cluster assuma uma nova trajetória, implicando em uma transformação na área de atuação do cluster (TRIPPL; OTTO, 2009).

Apesar da importância da introdução de novas rotinas durante o processo de renovação, todo o processo de renovação será impactado pela trajetória histórica do cluster (BOSCHMA, 2015; TREADO; 2010). Novas trajetórias não emergem em um modelo de "regiões virgens", mas sim através de um processo de recombinação de velhas e novas rotinas (MARTIN, 2010). O passado histórico de um cluster tanto limitará a sua capacidade de renovação, como também estipulará as novas oportunidades (BOSCHMA, 2015). O efeito lock-in explica apenas o processo de declínio dos clusters através das dinâmicas internas que corroem a sua capacidade de adaptação, explicando muito pouco como os clusters reagem a choques externos (ØSTERGAARD; PARK, 2013; MARTIN; SUNLEY, 2015). Desta forma, a ideia de resiliência de cluster visa preencher este vazio, explicando como os clusters reagem a choques e se adaptam a eles.

\section{Resiliência: um conceito em construção}

Resiliência significa "voltar para trás" e dentro da literatura social e ecológica está relacionada com a habilidade de um material absorver ou suportar mudanças infligidas no sistema a partir de fontes externas (HOLM; ØSTERGAARD, 2015). Nesse sentido, a resiliência reflete a ideia de adaptabilidade e sobrevivência de um sistema. Entretanto, o termo vem sendo utilizado em diferentes disciplinas, tais como psicologia, ecologia, engenharia, estudos em desastres, economia e geografia. A interpretação do termo resiliência é normalmente é dividida em três grandes grupos de definições, sendo eles: resiliência física, resiliência ecológica e resiliência adaptativa (MARTIN; SUNLEY, 2015; BOSCHMA, 2015).

A resiliência física é a mais restritiva e está relacionada com a ideia recuperar a forma e posição elástica de um sistema após um distúrbio (MARTIN; SUNLEY, 2015). O foco é na resistência que um sistema possui e a velocidade que o mesmo retorna ao seu estado anterior à ocorrência do choque (MARTIN, 2012). A resiliência física assume que um sistema se encontra em um estado de equilíbrio e a resiliência é definida em termos de estabilidade do sistema (HOLLING, 1973; 2001; MARTIN, 2012). Nesse sentido, um sistema é mais resiliente quando é menos vulnerável ou mais resistente e retorna, de forma rápida, ao seu estado inicial. A resiliência ecológica foi introduzida por Holling (1973) e se refere a uma propriedade que determina a persistência das relações dentro de um sistema ecológico, sendo uma medida de mensuração da capacidade dos sistemas de absorver as mudanças e persistir (HOLLING, 1973).

A resiliência ecológica refere-se ao choque que faz com que o sistema se mova para um outro regime de comportamento (SIMMIE; MARTIN, 2010). A resiliência é então entendida como a capacidade de um sistema de absorver um choque sem mudar a sua estrutura, identidade e funcionamento (MARTIN; SUNLEY, 2015). Se um choque excede a capacidade de absorção do sistema, então o sistema será empurrado para outro estado de equilíbrio (MARTIN; SUNLEY, 2015). Apesar de ser mais dinâmica do que a resiliência física, a ideia de resiliência ecológica não rompe com a ideia de equilíbrio (BOSCHMA, 2015; MARTIN, 2012). A perspectiva da resiliência ecológica impõe que uma região mudaria sua estrutura e funcionamento frente a um choque externo e se moveria para um novo estado de equilíbrio 
(BOSCHMA, 2015). Devido ao fato de que a economia é um processo histórico e contingente, é impossível ser conciliada a ideia de equilíbrio (MARTIN; SUNLEY, 2015).

A resiliência adaptativa discute a capacidade de um sistema de manter o seu desempenho, adaptando a sua estrutura, função e organização frente a um choque (MARTIN; SUNLEY, 2015). Dentro dos estudos regionais, a abordagem adaptativa foca na evolução das regiões e sua habilidade de se adaptar e reconfigurar suas bases industriais, tecnológicas e institucionais dentro de um sistema econômico o qual está constantemente evoluindo (BOSCHMA, 2015). Nesse sentido, essa resiliência se mostra mais como um processo em andamento e dinâmico do que uma recuperação de um estado de equilíbrio (MARTIN, 2012). A resiliência adaptativa regional inclui a habilidade de uma região de resistir a um choque, a habilidade de fazer pequenas ou grandes mudanças, se recuperar, se reorientar e a habilidade de se transformar completamente (HOLM; ØSTERGAARD, 2015). Para uma região ser considerada resiliente é necessário que ela mantenha níveis aceitáveis de crescimento, empregabilidade e riqueza à medida que se modifica (MARTIN, 2012). Ainda, Martin (2012) sugere quatro dimensões que descrevem como as regiões reagem aos choques. A primeira é a resistência, ou seja, a vulnerabilidade de uma economia regional; a segunda dimensão é velocidade e a extensão de recuperação frente ao choque; a terceira dimensão se refere à extensão na qual uma economia regional sofre uma reorientação estrutural; a quarta dimensão se refere ao grau de renovação ou retomada de uma trajetória de crescimento (MARTIN, 2012).

\section{Resiliência de cluster}

O crescente interesse sobre resiliência também se expandiu para os clusters (WROBEL, 2013; ØSTERGAARD; PARK, 2013; HOLM; ØSTERGAARD, 2015). O primeiro passo para a elaboração de um framework sólido de resiliência de cluster começa através da elaboração de um conceito que consiga transpor a ideia de resiliência econômica para os clusters. Cabe ressaltar que a resiliência de cluster é um conceito populacional (HOLM; ØSTERGAARD, 2015), isto significa que nem todas as firmas sobreviverão a um choque. Østergaard e Park (2013) elaboraram a definição mais popular de resiliência de cluster, definindo a resiliência de cluster como "uma capacidade adaptativa que permite com que o cluster faça mudanças para superar disrupções internas e externas e ainda continuar funcionando com a sua identidade como um cluster dentro de um campo particular" (ØSTERGAARD; PARK, 2013, p.2).

O principal problema da definição de Østergaard e Park (2013) é a limitação da resiliência em manter uma identidade do cluster dentro de um campo em particular. Essa perspectiva é fortemente enraizada na definição de cluster de Porter (1998), na qual a identidade do cluster está atrelada ao seu setor. A perspectiva porteriana é uma ideia estática de cluster, uma vez que pouca ou nenhuma transformação ocorre. Staber e Sautter (2011) descrevem a identidade do cluster como o compartilhamento da base industrial, tecnológica, social e de características institucionais do cluster. A identidade do cluster não é estática, mas que se desenvolve e se transforma ao longo do tempo, não estando relacionada apenas com o setor de atuação, mas também com outras características sociais e institucionais que se enraízam à medida que o cluster se desenvolve (STABER; SAUTTER, 2011). Nesse sentido, a identidade do cluster se modifica ao longo do tempo, uma vez que o cluster pode se renovar para setores relacionados, o que manteria a identidade setorial intacta, mas também para setores não-relacionados, o que modificaria a sua identidade setorial (MARTIN; SUNLEY, 2011).

Ao invés de assumir a identidade do cluster somente através de uma identidade setorial, diversos trabalhos passaram a assumir as networks como principal componente da identidade do cluster (BRAMWELL et al., 2008; STABER; SAUTTER, 2011; CRESPO et al., 2014). A identidade do cluster através das redes que o compõe permite a compreensão do cluster como um sistema adaptativo complexo (MARTIN; SUNLEY, 2007) que se transforma ao longo dos anos, à medida que seus agentes se adaptam as novas realidades econômicas. Desta forma, com base nos trabalhos de Boschma (2015) e Martin e Sunley (2015), a definição de resiliência de cluster adotada neste trabalho é a capacidade de um cluster em resistir ou se recuperar de um choque, reconfigurando as suas bases tecnológicas, sociais e institucionais, através de mudanças adaptativas 
na sua estrutura, ou ainda, se redirecionar para novas trajetórias não-relacionadas, permitindo assim com que o cluster atinja niveis produtivos maiores ou semelhantes ao periodo antecessor ao choque.

Este conceito de resiliência de cluster permite o entendimento do cluster como um sistema complexo que se transforma ao longo do tempo. Um cluster será considerado resiliente quando não for significativamente afetado por um choque. Nesse sentido, existe uma enorme quantidade de possíveis choques que um cluster poderá enfrentar: econômico, político-institucional, tecnológico, setorial, etc. Ainda, um cluster pode resistir a um choque específico, mas ser vulnerável a outros choques. Desta forma, a vulnerabilidade estará tanto relacionada à natureza do choque, como também as características internas do cluster. A segunda parte da definição está relacionada à recuperação do cluster após o choque. A recuperação não está apenas atrelada à velocidade com que o cluster retorna as suas características iniciais anteriores ao choque, uma visão adaptativa faz reconhecer que a resiliência é um processo recursivo no qual o choque e a sua recuperação estão entrelaçados (MARTIN; SUNLEY, 2015) e que influenciarão permanentemente a trajetória futura do cluster (MARTIN, 2012; SIMMIE; MARTIN, 2010).

A forma como os clusters lidam e se transformam perante às mudanças não são homogêneas. Entretanto, uma característica comum do processo de recuperação dos cluster frente aos choques é a mudança nas bases tecnológicas, sociais e institucionais. Diversos estudos demonstram o processo de recuperação de clusters após choques, demonstrando que os clusters conseguem se recuperar reajustando suas estruturas, através de mudanças adaptativas (TREADO, 2010; HASSINK, 2010; HERVAS-OLIVER et al., 2011), mas também através de profundas mudanças estruturais (COENEN et al., 2013; TRIPPL; OTTO, 2009). Em ambos os processos de recuperação, os clusters foram permanentemente transformados pelo seu processo de recuperação. Assim como argumentado por Holm e Østergaard (2015), a resiliência de cluster envolve a adaptação e também inclui a capacidade do cluster em resistir ao choque e fazer pequenas ou grandes mudanças que reorientem ou que o transformem.

\section{Clusters e o modelo de panarquia}

A conceptualização da resiliência como a capacidade de um cluster em se adaptar em resposta aos choques reflete diretamente com a ideia de sistemas adaptativos complexos (SAC) (MARTIN; SUNLEY, 2015; BRISTOW; HEALY, 2014). A abordagem dos SAC's reconhece que os sistemas são caracterizados por dinâmicas complexas, não-lineares e que ocorrem dentro de um contexto de incerteza e mudanças, não estando nunca em equilíbrio (BRISTOW; HEALY, 2014). Tal abordagem também procura entender os padrões e as inter-relações dentro de um sistema ao invés de simples dinâmicas de causa e efeito (BRISTOW; HEALY, 2014). A auto-organização de um SAC permite com que o mesmo adapte as suas estruturas de acordo com as mudanças externas que ocorrem em um ambiente (SIMIE; MARTIN, 2010). Ainda, mesmo que algumas partes do sistema não sobrevivam às mudanças, o sistema continua existindo (MARTIN; SUNLEY, 2015).

Dentro da perspectiva dos SAC's, os agentes adaptam o seu comportamento de forma continua, com base nas suas observações do sistema e de outros ao seu redor, através de mecanismos de aprendizado (BRISTOW; HEALY, 2014). Desta forma, à medida que os agentes adquirem conhecimento, eles modificam as suas estruturas para melhor se adequarem às mudanças que ocorrem no seu ambiente (BRISTOW; HEALY, 2014). Apesar de serem dependentes de sua trajetória histórica, os SAC's podem produzir inúmeras trajetórias distintas e mudanças imprevisíveis devido as suas múltiplas interações e auto-organização (MARTIN; SUNLEY, 2011). Desta forma, a resiliência, dentro do campo da teoria de sistemas, é compreendida pela forma na qual os sistemas complexos conseguem se antecipar ou se reorganizar após um determinado choque (EVANS; KARECHA, 2014). A partir da ideia de que clusters se enquadram no framework dos SAC's, diversos autores passaram a utilizar o modelo de panarquia para expressar o modelo de transformação dos clusters, focando no trade-off entre a estabilidade e mudança (SIMIE; MARTIN, 2010). 
O modelo de panarquia consiste de quatro fases que caracterizam o sistema através de processos contínuos de ajustes. As três principais características que variam em cada fase são: o potencial acumulado de recursos disponíveis no sistema; a conexão interna dos atores e componentes que compõem o sistema; e a resiliência, uma mensuração da vulnerabilidade e recuperação do sistema (SIMMIE; MARTIN, 2010; MARTIN; SUNLEY, 2011). O primeiro estágio é chamado de "exploração" (exploitation) ou " $\mathrm{r}$ " e se caracteriza por uma rápida acumulação de recursos. Esta é a fase na qual o sistema emerge e se desenvolve. À medida que o cluster começa a se desenvolver, ele acumula rotinas que se difundem rapidamente através das redes, permitindo um maior desenvolvimento econômico regional. A segunda fase é chamada de conservação ou " $k$ " e é caracterizada pelo aumento da estabilidade e rigidez do sistema. Nesta fase, o grau de conexão é alto, mas com pouco espaço para a inovação e resiliência, uma vez que o sistema tende a se auto reproduzir. A conexão entre os componentes do cluster aumenta devido ao mecanismo de ligação preferencial e a emergência de um design dominante torna o cluster rígido.

Caso ocorra um choque, o sistema entra na terceira fase chamada de "libertação" ou " $\Omega$ ". Nesta fase, o sistema declina, diminuindo a sua conexão, mas também abre espaço para a inovação e a reestruturação. A fase de libertação é constituída pelo declínio do cluster. Entretanto, esta fase também reduz as barreiras estipuladas pela fase anterior, permitindo que as firmas sobreviventes rompam com a trajetória dominante e busquem por inovações. Desta forma, o sistema entra na quarta fase chamada de "reorganização" ou " $\alpha$ ". Nesta fase, a resiliência volta a aumentar devido à exploração múltiplas trajetórias que dão origem a novas atividades, novas tecnologias e novas vantagens competitivas que renovarão as bases do cluster (MARTIN; SUNLEY, 2011; SIMMIE; MARTIN, 2010; PENDAL et al., 2010).

O modelo de panarquia sugere que a mudança não é iminente e pré-determinada, ao invés disto, ela é imprevisível devido ao resultado das interações e das novas experimentações (MARTIN; SUNLEY, 2011). Dentro do modelo de panarquia, a resiliência (adaptabilidade) e acumulação (adaptação) são duas propriedades que estão em constante evolução. Apesar de relevante para se entender o processo de evolução dos clusters, o modelo de panarquia só funciona para analisar a trajetória de forma retrospectiva, não sendo adequado para prever trajetórias futuras.

\section{Elementos da Resiliência de um Cluster}

Os clusters são estruturas de redes complexas que estão inter-relacionadas através de relações sociais e comerciais. Para uma análise mais precisa dos elementos que tornam essas redes mais ou menos resilientes, tanto os elementos internos, como os externos devem ser levados em consideração, bem como a trajetória histórica do cluster. Os clusters são estruturas econômicas que estão em constante transformação. Desta forma, os elementos que compõem a sua resiliência também possuem um caráter evolucionário, se modificando e adaptando, de forma recursiva, à medida que o cluster evolui. A Figura 1 apresenta o framework para resiliência de clusters proposto neste trabalho.

O ponto de partida de análise é a estrutura tecnoindustrial na qual o cluster está inserido e que lhe renderá externalidades de localização. Clusters são definidos como estruturas econômicas altamente especializadas em um setor. Tal especialização torna a região mais vulnerável a um choque em relação a regiões diversificadas (HOLM; ØSTERGAARD, 2015). Apesar de serem mais vulneráveis aos choques setoriais, os clusters tendem a obter índices de desempenho superiores, através da geração de externalidades marshallianas voltadas à alta especialização e à produção (FRENKEN et al., 2007; NEFFKE et al., 2011). A alta especialização inicialmente facilita o desenvolvimento econômico, mas aprisiona o cluster em uma trajetória. Desta forma, o grande problema dos clusters, principalmente aqueles localizados em regiões com pouca diversidade econômica, é a baixa diversidade de conhecimentos (TÖDTLING; TRIPPL, 2005), o que leva o cluster a uma estrutura homogênea que reduz novas combinações de recursos e conhecimentos (SUIRE; VICENTE, 2014). 


\section{Figura 1 - Framework da resiliência de clusters}

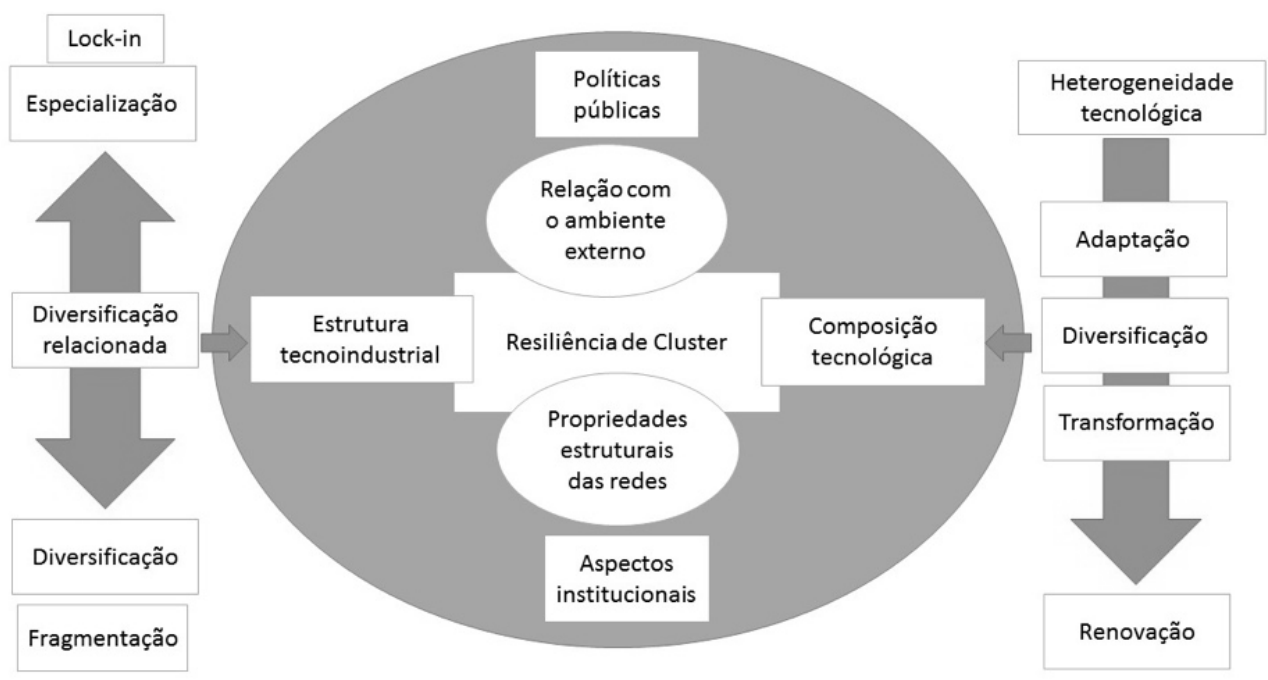

Já os clusters inseridos em regiões diversificadas identifica-se externalidades jacobianas, as quais se referem a novas combinações de atividades econômicas entre diferentes setores, diversificação essa que auxilia na inovação, à medida que diferentes conhecimentos transbordam e são recombinados (FARHAUER; KROLL, 2012). Entretanto, as regiões altamente diversificadas podem sofrer da falta de foco, falta de massa crítica, falta de proximidade cognitiva e coerência tecnológica (BOSCHMA, 2015). Desta forma, tais regiões correm o risco de se fragmentarem (TÖDTLING; TRIPPL, 2005). Neste sentido, enquanto as externalidades marshallianas são geradas através da especialização e produzem benefícios para a produção, as externalidades jacobianas são geradas através da diversificação e aumentam a probabilidade da geração de inovações.

O trade-off entre especialização e diversificação é antigo e foi descrito por Grabher (1993) na distinção de adaptação e adaptabilidade. Grabher (1993) notou que, à medida que uma região se torna altamente produtiva devido à adaptação de suas estruturas, a mesma tende a perder a sua capacidade de explorar novas trajetórias, denominando este processo de "armadilha da especialização". Para que um cluster seja considerado resiliente, ele deve ser capaz de superar a tensão entre adaptação e adaptabilidade, ou seja, conseguir atingir altos níveis de produtividade, mas sem sacrificar a sua capacidade de adaptação no longo prazo (BOSCHMA, 2015). Nesse sentido, diversos autores passaram a defender o papel da variedade relacionada (BOSCHMA, 2015; FRENKEN et al., 2007; NEFFKE et al., 2011).

A variedade relacionada se refere à ideia de que uma região pode possuir uma estrutura especializada, mas com setores tecnologicamente relacionados, os quais aumentam o potencial de aprendizagem, através de novas combinações entre os setores (FRENKEN et al., 2007). A grande vantagem das firmas localizadas em regiões que possuem uma estrutura econômica especializada, mas ao mesmo tempo tecnologicamente relacionada com outros setores, é que as firmas podem explorar tanto as externalidades de especialização, como de diversificação (FARHAUER; KROLL, 2012). Setores relacionados compartilham a mesma base de conhecimentos, desta forma, inovações em um setor podem ser aproveitadas por outros setores relacionados (FRENKEN et al., 2007). Kahl e Hundt (2015) descobriram que a especialização em uma rede possibilita que as firmas cresçam mais rápido, mas também as tornam mais suscetíveis aos choques. Já as firmas inseridas em redes mais diversificadas conseguem manter melhores resultados econômicos após um choque (KAHL; HUNDT, 2015). Desta forma, a estruturação de uma rede no formato de cluster em uma região com uma economia diversificada conseguiria se desenvolver rapidamente, sem perder a sua adaptabilidade. 
Além da capacidade de resistir a choques, a resiliência também se refere na capacidade de assumir novas trajetórias de crescimento. Nesse sentido, novos setores tendem a emergir a partir de setores que são tecnologicamente relacionados às indústrias existentes. Neffke e Henning (2013) descobriram que a diversificação é 123 vezes mais provável de ocorrer em setores que possuem laços com atividades centrais das firmas do que setores que não possuem. Além disto, setores relacionados também conseguem manter níveis de empregabilidade maiores após uma choque, uma vez que os trabalhadores buscam trabalhar em áreas que necessitem de conhecimentos já desenvolvidos por eles (NEFFKE; HENNING, 2013). Ainda, os setores tecnologicamente semelhantes também possuem uma maior probabilidade de sobrevivência em comparação com os setores tecnologicamente periféricos (NEFFKE et al., 2011).

A resiliência do cluster também depende das propriedades estruturais da rede de conhecimento (BOSCHMA, 2015; SUIRE; VICENTE, 2014; CRESPO et al., 2014). A medida que as firmas possuem diferentes níveis de conhecimentos (BOSCHMA; FRENKEN, 2006), a criação de novos conhecimentos e o aprendizado local depende da combinação de diferentes conhecimentos presentes nas redes (BOSCHMA, 2015; NOOTEBOOM, 2000). Ainda, as redes também possuem um trade-off entre conectividade e resiliência (SIMMIE; MARTIN, 2010). Se por um lado existe a tendência dos sistemas em aumentar a sua conectividade, a fim de se aumentar a eficiência, através da proximidade dos aspectos sociais, cognitivos, organizacionais e institucionais (BOSCHMA, 2005), o aumento desta conectividade reduz a sua adaptabilidade. Quanto mais conectado for um sistema, mais rígido e menos adaptável ele tende a ser (SIMMIE; MARTIN, 2010). O conflito entre conectividade e adaptabilidade das redes é acomodado pelo modelo de panarquia, o qual aplica uma perspectiva dinâmica na qual os níveis de resiliência se modificam de forma contínua, passando por fases de maior rigidez e fases com maior flexibilidade (SIMMIE; MARTIN, 2010).

O trade-off entre adaptação e adaptabilidade pode ser superado a partir da análise das propriedades das redes (BOSCHMA, 2015; SUIRE; VICENTE, 2014; CRESPO et al., 2014). Nesse sentido, Suire e Vincente (2014) e Crespo et al. (2014) propõem uma avaliação das relações das redes em termos de relações de centro-periferia. Dentro deste modelo, algumas firmas concentram a maior parte dos laços e da informação, devido ao mecanismo de ligação preferencial. Essas firmas centrais passam a difundir conhecimentos, conformidade e estabelecer as normas sociais entre os membros da rede (SUIRE; VINCENTE, 2014). As firmas centrais se tornam difusoras de um design dominante, estabelecendo uma maior estabilidade para a rede. Já as firmas pertencentes às periferias permanecem menos enraizadas na rede, mantendo fortes relações com o mundo exterior e se tornam fontes preciosas de novos conhecimentos (SUIRE; VICENTE, 2014). Desta forma, o cluster deve preservar uma coesão interna no centro, à qual será responsável pela adaptação, enquanto multiplica os canais de acesso a novos conhecimentos na periferia, permitindo a entrada de novos conhecimentos que garantem a sua adaptabilidade (CRESPO et al., 2014).

Boschma (2015) ainda analisa a natureza das relações da rede em termos de proximidade entre os agentes. O framework sobre proximidade desenvolvido por Boschma (2005) estabelece que a proximidade entre os agentes favorece a formação de redes, uma vez que a proximidade diminuiu os custos e os riscos, entretanto muita proximidade pode levar a rede ao aprisionamento (BOSCHMA, 2015; BOSCHMA, 2005). A noção de proximidade cognitiva impõe que as firmas que compartilham as mesmas bases tecnológicas aprendem mais umas com as outras do que firmas com bases muito distantes (FRENKEN et al., 2007; NOTEBOOM, 2000). Desta forma, para que haja um efetivo transbordamento de conhecimentos, é necessário que exista uma proximidade cognitiva entre as firmas que permita com que o conhecimento seja acessado e explorado (NOOTEBOOM, 2000). Entretanto, uma distância cognitiva muito curta torna o conhecimento redundante e pouco valorizado, podendo levar a região ao lock-in negativo, bem como ao acirramento da competição, dificultando as atividades colaborativas (BOSCHMA, 2015).

De forma relacionada com as propriedades das redes, as relações do cluster com o ambiente externo também impacta na sua resiliência. Grande parte dos estudos sobre clusters focam no impacto das configurações 
locais no desempenho das firmas, ignorando as interconexões externas que as firmas clusterizadas mantém e o impacto que estas conexões possuem no cluster (BATHELT et al., 2004; HERVAS-OLIVER et al., 2011; BRAMWELL et al., 2008; SUIRE; VICENTE, 2014). Ainda que os clusters sejam aglomerados geográficos de empresas e instituições, as suas relações de troca e de acesso a novos conhecimentos não se limitam ao nível regional (BATHELT et al., 2004). De fato, os clusters podem se beneficiar da sua inserção na cadeia global de valor, o que permite o acesso a novos mercados e fontes de conhecimentos (BATHELT et al., 2004), os quais podem ser transmitidos através das relações centroperiferia. Entretanto, para que a relação centro-periferia torne a rede mais resiliente, é necessário que as firmas possuam uma alta capacidade absortiva, a fim de absorver os conhecimentos fora da rede, assimilálos e difundi-los dentro da rede (MENZEL; FORNAHL, 2010).

Suire e Vicente (2014) ainda destacam o fato de que o estágio do ciclo de vida da tecnologia utilizada no cluster é um fator que impacta na resiliência do cluster. Se o declínio na demanda de mercado para uma dada tecnologia for considerada um choque, alguns clusters podem demonstrar uma maior habilidade para prolongar o ciclo tecnológico, estendendo o escopo de atuação para outros campos (SUIRE; VICENTE, 2014).

Nos últimos anos, a ideia de ciclo de vida de cluster se tornou bastante popular (MENZEL; FORNAHL, 2010; MARTIN; SUNLEY, 2011), tal abordagem teve como origem a mesma metáfora utilizada para descrever o ciclo de vida de produtos, de tecnologias e indústrias (KLEPPER, 1997). Klepper (1997) identificou três principais estágios característicos para o ciclo de vida das indústrias. Nos estágios iniciais, o volume de mercado é baixo, a incerteza é alta e o design do produto é primitivo. Nesta fase, as firmas exploram (exploration) diversas possibilidades tecnológicas. Já nos estágios mais avançados, o crescimento da produção aumenta, o design dos produtos tende a se estabilizar e a taxa de inovação diminui. Neste estágio, as firmas tornam a sua exploração (exploitation) mais direcionada para uma tecnologia mais específica, homogeneizando as suas rotinas.

A viabilidade e o desempenho competitivo de um cluster depende da sua habilidade de estabelecer padrões tecnológicos que superem seus concorrentes (SUIRE; VICENTE, 2014). Os clusters serão mais resilientes se conseguirem combinar a eficiência da exploração (exploitation) de sua fase madura, mantendo uma pequena parcela de exploração (exploration) característica de suas fases iniciais (SUIRE; VICENTE, 2014). Desta forma, caso um choque diminua a demanda pela tecnologia do cluster, ele será mais capaz de se readaptar a novas necessidades. Aqui, o cluster poderá seguir o processo de renovação descrito por Trippl e Otto (2009). Além disto, a resiliência também dependerá do campo tecnológico no qual o cluster está envolvido (SUIRE; VICENTE, 2014). Nesse sentido, setores emergentes ou de alta tecnologia possuem uma maior probabilidade de renovação (MARTIN; SUNLEY, 2015).

Outro elemento importante é o papel das instituições na resiliência do cluster (BOSCHMA, 2015). As instituições estão interligadas a diversas dimensões, uma vez que as instituições facilitam ou não as interações entre as bases de conhecimento e industriais dentro de uma região (BOSCHMA, 2015). Os aspectos institucionais podem ter um grande efeito na capacidade adaptativa de uma região (BRISTOW; HEALY, 2014). Saxenian (1995) comparou a trajetória de recuperação dos cluster de circuitos eletrônicos do Vale do Silício e da Rota 128 após o choque de demanda. O que foi determinante para essa renovação do cluster do Vale do Silício foi o fato de que o cluster possuía uma propensão à inovação muito mais forte, uma vez que nele havia uma cultura local que estimulava a cooperação e a diversificação (SAXENIAN, 1995). O exemplo trazido por Saxenian (1995) indica que as dinâmicas institucionais são moldadas e limitadas por um conjunto de normas e hábitos culturalmente enraizados e que impactam na capacidade adaptativa de uma região.

Ao passo que novos clusters emergem e se desenvolvem, as instituições tendem a co-evoluir junto com as transformações que ocorrem (COENEN et al., 2013). Conforme uma região se especializa em um setor, a estrutura institucional daquela região tende a se adequar as necessidades específicas daquele setor (BOSCHMA, 2015). Entretanto, tal adaptação pode desestimular a emergência de outros setores que não receberam aporte e visibilidade institucional. Tal aspecto foi descrito por Grabher (1993) como lock-in 
político e se refere as fortes relações institucionais que objetivam preservar as estruturas econômicas existentes, mesmo quando são ineficientes (GRABHER, 1993; BRISTOW; HEALY, 2014). Desta forma, é possível supor que uma estrutura econômica mais variada tende a fornecer um quadro institucional mais adaptável, uma vez que, dificilmente, algum agente conseguirá dominar o quadro institucional (BOSCHMA, 2015). Entretanto, tais regiões muito diversificadas também possuem um problema com a coesão institucional local, uma vez que muitos interesses prejudicam o foco, a coordenação e o controle institucional (BOSCHMA, 2015).

Boschma (2015) propõe a ideia de que o conflito de interesses institucionais seria superado quando os setores e as bases de conhecimento fossem complementários ou sobrepostos institucionalmente. Nesta direção, Grillitsch (2014) cunhou o termo "camadas institucionais". De acordo com o autor, as camadas institucionais são o conjunto de regras e restrições que governam a interação entre indivíduos que pertencem a uma estrutura social distinta (GRILLITSCH, 2014, p. 7). Nesta abordagem, o quadro institucional de uma região é composto pelas camadas institucionais nele presente e impactam o desempenho daquela região. Regiões que compartilham camadas institucionais semelhantes tendem a aumentar a sua coesão regional e alcançar maiores níveis de resiliência devido à criação de um ambiente que facilita a transferência de conhecimentos e um ambiente institucional coerente que consegue suportar as demandas regionais e se adaptar a elas (GRILLITSCH, 2014). Assim, uma região que possuísse complementariedades institucionais seria melhor equipada para explorar novas recombinações entre os setores (GRILLITSCH, 2014).

Uma das principais razões pelas quais a resiliência se tornou tão popular é devido ao seu apelo para formulação de políticas públicas que influenciem na prevenção, recuperação e criação de novas trajetórias após um choque. A importância da inovação para o desempenho econômico já é reconhecida pelas firmas e pelos agentes políticos (TÖDTLING; TRIPPL, 2005; ERAYDIN, 2016). O interesse político se estendeu para os estudos regionais, uma vez que organizações de suporte aos clusters tendem a emergir a fim de facilitar o crescimento econômico e defender os interesses das firmas (TREADO, 2010). Entretanto, a agenda política tendia a replicar modelos de sucesso, ignorando especificidades econômicas e institucionais locais (TÖDTLING; TRIPPL, 2005). O fracasso de diversos projetos políticos de desenvolvimento e renovação de clusters demonstrou que não existe um modelo ideal de política regional. Tödtling e Trippl (2005) sugerem uma tipologia baseada em três diferentes tipos de regiões: periféricas, regiões antigas e metrópoles. Visando à renovação de clusters em regiões periféricas, os autores sugerem políticas públicas voltadas para a renovação e introdução de novos campos tecnológicos e atração de novas empresas (TÖDTLING; TRIPPL, 2005). Desta forma, os agentes políticos podem tornar um cluster mais robusto e mais adaptativo aos choques, através da diversificação das bases tecnológicas regionais (MARTIN, 2012). Outros elementos podem ser estimulados por políticas públicas e impactam na resiliência (ERAYDIN, 2016), tais como a qualificação do capital humano (TREADO, 2010), existência de recursos financeiros (CRISTOPHERSON et al., 2010), estímulos ao empreendedorismo (BRAMWELL et al., 2008), qualificação da infraestrutura pública (EVANS; KARECHA, 2014) e manutenção de relacionamentos com outros clusters e polos inovativos (HERVASOLIVER et al., 2011).

Em suma, o framework desenvolvido apresenta diversos elementos importantes para o desenvolvimento e sustentação de um cluster. Contudo, a mera presença de um ou mais elementos não é suficiente para assegurar a resiliência, uma vez que a resiliência é um constructo que leva em consideração a combinação de diversos aspectos. A evolução do cluster é um processo recursivo no qual o cluster molda e é moldado pelo ambiente, isto faz com que cada componente deste framework se modifique constantemente. 


\section{Considerações Finais}

O fenômeno de aglomerações geográficas de empresas vem atraindo a atenção de pesquisadores desde o final do século XIX com o trabalho seminal de Marshall, em 1890. Na década de 1970, o interesse nessas aglomerações ressurgiu, especialmente associado ao desenvolvimento regional. Entretanto, foi somente após a aplicação da economia evolucionária nos estudos regionais (BOSCHMA; FRENKEN, 2006) que questões relacionadas à dinâmica dos clusters começaram a ser discutidas (SUIRE; VICENTE, 2014; MARTIN; SUNLEY, 2011; HOLM; ØSTERGAARD, 2015). Nesse contexto, os clusters não são considerados como estruturas econômicas estáticas, que garantem o crescimento regional somente devido à geração de externalidades (MARTIN; SUNLEY, 2006). A visão estática, presente principalmente nos trabalhos de Porter sobre cluster (PORTER, 1998), não é capaz de explicar os processos evolucionários que caracterizam o seu desenvolvimento. Diante disso, neste ensaio teórico, recorreu-se a abordagem de sistemas complexos adaptativos (MARTIN; SUNLEY, 2007), uma vez que tal perspectiva rejeita a ideia de equilíbrio e trata os clusters como uma forte rede de agentes que compõem um sistema, o qual está em constante transformação (BRAMWELL et al., 2008; CRESPO et al., 2014).

Nessa trajetória dinâmica, a resiliência dos clusters torna-se um elemento de crescimento interesse diante das sucessivas crises globais. Apesar da sua relevância, poucos estudos investigaram empiricamente a resiliência de clusters. Wrobel (2013) realizou um estudo na Alemanha comparando firmas dentro e fora de clusters. Os resultados demonstraram que as firmas em cluster eram mais resistentes diante de choques econômicos, mas o tema ainda carece de investigações, especialmente no contexto de países emergentes.

Como principais contribuições teóricas, este artigo redefiniu resiliência de cluster com base nos trabalhos de Boschma (2015) e Martin e Sunley (2015). A definição utilizada aqui está alinhada com a literatura evolucionária, uma vez que leva em consideração a resistência e a recuperação de um cluster após um choque, bem como o seu redirecionamento para novas trajetórias não-relacionadas com o seu setor de atuação. A resiliência trata de como os clusters conseguem adaptar suas bases tecnológicas e capacidades locais a fim de explorar comercialmente novas fontes de conhecimentos (WOLFE, 2010). Neste sentido, a resiliência de um cluster está diretamente ligada com a sua capacidade de resistir e se recuperar de choques, mas também de criar novos conhecimentos que permitam a exploração econômica de novas oportunidades (BALLAND; RIGBY; BOSCHMA, 2015). Com base nessa definição, o framework proposto neste artigo considera os principais elementos que podem afetar a capacidade de um cluster de resistir, se recuperar e assumir novas trajetórias. Os elementos citados influenciam principalmente a estrutura de aquisição e recombinação de novos conhecimentos do cluster, destacando tanto os aspectos internos, como os externos ao cluster.

A resiliência é um constructo que leva em consideração tantos os elementos econômicos e nãoeconômicos (ÖZ; ÖZKARACALAR, 2011) e o sucesso do cluster dependerá da forma como eles se recombinam frente aos choques. A mera presença de um ou mais elementos não é suficiente para assegurar a resiliência de um cluster, uma vez que o choque pode afetar um ponto do cluster de maior fragilidade. Mantendo o caráter evolucionário, tais elementos podem se modificar ao longo do tempo (DIODATO; WETERINGS, 2012). Nesse sentido, um cluster pode dispor de uma alta resiliência em um determinado período, mas perder tal capacidade ao longo do tempo. Isso ocorre porque a evolução do cluster é um processo recursivo no qual o cluster molda e é moldado pelo ambiente, isto faz com que cada componente deste framework se modifique constantemente ao longo da trajetória do cluster.

A discussão sobre resiliência vem acompanhada do trade-off entre adaptação e adaptabilidade (BOSCHMA, 2015; MARTIN; SUNLEY, 2015). De um lado, estruturas econômicas muito especializadas se tornam mais competitivas, mas tendem a ser mais vulneráveis a choques e a perderem a sua capacidade de adaptação. Por outro lado, estruturas econômicas muito diversificadas tendem a ser mais inovativas, mas correm o risco de não serem competitivas e de se fragmentarem. Como contribuição empírica, uma possível solução para este trade-off está relacionada com a manutenção de uma estrutura econômica-social-institucional coesa, mas com diversos agentes relacionados que consigam aproveitar 
tanto os conhecimentos gerados no cluster, como também inserir novos conhecimentos. As externalidades geradas pelas firmas centrais do cluster mantém ele produtivo e coeso, enquanto as firmas periféricas e relacionadas irrigam o cluster com novos conhecimentos que permitem com que o cluster se renove e passe a explorar novos mercados (SUIRE; VICENTE, 2014).

Por fim, este trabalho trata-se de um ensaio teórico e novos artigos poderão desenvolver pesquisas empíricas para analisar a influência de cada um desses elementos propostos. Cabe ainda destacar que a recente crise na economia brasileira (OREIRO, 2017) torna a discussão sobre resiliência de cluster bastante oportuna e oferece oportunidades empíricas de investigação sobre como os clusters enfrentam choques.

\section{Referências}

BALLAND, P. A.; RIGBY, D.; BOSCHMA, R. The technological resilience of US cities. Cambridge Journal of Regions, Economy and Society, V. 8, No. 2, p. 167-184, 2015.

BATHELT, H.; MALMBERG, A.; MASKELL, P. Clusters and knowledge: Local buzz, global pipelines and the process of knowledge creation, Progress in Human Geography, V. 28, №. 1, p. 31-56, 2004.

BOSCHMA, R. A. Proximity and Innovation: A Critical Assessment, Regional Studies, Vol. 39, №. 1, p.61-74, 2005.

BOSCHMA, R. A. Towards an evolutionary perspective on regional resilience. Papers in Evolutionary Economic Geography, V. 9, p. 1-27, 2015.

BOSCHMA, R. A.; FRENKEN, K. Why is economic geography not na evolutionary Science? Towards na evolutionary economic geography, Journal of Economic Geography, V. 6, p. 273302, 2006.

BRAMWELL, A.; NELLES, J.; WOLFE, D. A. Knowledge, Innovation and Institutions: Global and Local Dimensions of the ICT Cluster in Waterloo, Canada, Regional Studies, V. 42, №. 1, p. 101116, 2008.

BRISTOW, G.; HEALY, A. Regional Resilience: An Agency Perspective, Regional Studies, V. 48, №. 5, 923-935, 2014.

COENEN, L.; MOODYSSON, J.; MARTIN, H. Renewal of Mature Industry in na old Industrial Region: Regional Innovation Policy and the Co-evolution of Institutions and Technology, Pappers in Innovation Studies 2013/7, CIRCLE, 2013.

CRESPO, J.; SUIRE, R.; VICENTE, J. Lock-in or lock-out? How structural properties of knowledge networks affect regional resilience, Journal of Economic Geography, V. 14, p. 199-219, 2014.

DIODATO, D.; WETERINGS, A. The Resilience of Dutch Regions to Economic Shocks, Papers in Evolutionary Economic Geography \# 12.15, 2012.

ERAYDIN, A. The role of regional policies along with the external and endogenous factors in the resilience of regions, Cambridge Journal of Regions, Economy and Society, V. 9, p. 217-234, 2016.

EVANS, R.; KARECHA, J. Staying on Top: Why is Munich so Resilient and Successful? European Planning Studies, V. 22, p. 1259 - 1279, 2014.

FARHAUER, O.; KROLL, A. Diversified specialisation - going one step beyond regional economics' specialisation-diversification concept, Jahrbuch für Regionalwissenschaft, V. 32, №. 1, p. 63-84, 2012. 
FRENKEN, R.; OORT, F. V.; VERBURG, T. Related Variety, Unrelated Variety and Regional Economic Growth, Regional Studies, V. 41, №. 5, p. 685-697, 2007.

GRABHER, G. The embedded firm: On the socioeconomics of industrial networks. London, New York: Routledge, pp. 256 - 277, 1993.

GRILLITSCH, M. Institutional Change and Economic Evolution in Regions, Working Paper Circle, Paper no. 2014/01, 2014

HASSINK, R. Locked in decline? On the role of regional lock-ins in old industrial áreas. In: Boschma, R.; Martin, R. (eds), Handbook of Evolutionary Economic Geography. Edward Elgar, Cheltenham, p. 450-468, 2010.

HERVAS-OLIVER, J-L.; JACKSON, I.; TOMLINSON, P. R. 'May the ovens never grow cold': regional resilience and industrial policy in the North Staffordshire ceramics industrial district - with lessons from Sassoulo and Castellon, Policy Studies, V. 32, №. 4, p. 377-395, 2011.

HOLLING, C. S. Resilience and stability of ecological systems, Annual Review of Ecology and Systematics, V. 4, p. 1-23, 1973.

HOLM, J. R.; ØSTERGAARD, C. R. Regional Employment Growth, Shocks and Regional Industrial Resilience: A Quantitative Analysis of the Danish ICT Sector, Regional Studies, V. 49, №. 1, p. 95-112, 2015.

KAHL, J.; HUNDT, C. Employment performance in times of crisis, Competitiveness Review, V. 25, №. 4, p. 371-391, 2015.

KLEPPER, S.; Industry Life Cycles, Industrial and Corporate Change, V. 6, №. 1, p. 145-181, 1997.

MARTIN, R. Roepke Lecture in Economic Geography - Rethinking Regional Path Dependence: Beyond Lock-in to Evolution, Economic Geography, Vol. 86, №. 1, p. 1-27, 2010.

MARTIN, R. Regional economic resilience, hysteresis and recessionary shocks. Journal of Economic Geography, V. 12, p. 1-32, 2012.

MARTIN, R.; SUNLEY, P. Path dependence and regional economic evolution. Journal of Economic Geography, V. 6, p. 395-437, 2006.

MARTIN, R.; SUNLEY, P. Complexity thinking and evolutionary economic geography, Journal of Economic Geography, V. 7, p. 603-618, 2007.

MARTIN, R.; SUNLEY, P. Conceptualizing Cluster Evolution: Beyond the Life Cycle Model? Regional Studies, V. 45, №. 10, pp. 1299-1318, 2011.

MARTIN, R.; SUNLEY, P. On the notion of regional economic resilience: conceptualization and explanation, Journal of Economic Geographic, V. 15, p. 1-42, 2015.

MENZEL, M. P.; FORNAHL, D. Cluster Life Cycles - Dimensions and Rationales of Cluster Evolution, Industrial and corporate Change, V.19, N.1, pp. 205-238, 2010.

MOROSINI, P. Industrial Clusters, Knowledge Integration and Performance. Word Development, V. 32, N. 2, pp. 305 - 326, 2004.

NEFFKE, F.; HENNING, M.; BOSCHMA, R. How do regions diversify over time? Industry relatedness and the development of new growth paths in regions, Economic Geography, V. 87, N. 3, p. 237-266, 2011.

NEFFKE, F.; HENNING, M. S. Skill-relatedness and firm diversification, Strategic Management Journal, V. 34, №. 3, p. 297-316, 2013. 
NOOTEBOOM, B. Learning and innovation in organizations and economies. Oxford, U. K.: Oxford University Press, 2000.

OREIRO, J. L. A grande recessão brasileiro: diagnóstico e uma agenda de política econômica, Estudos Avançados, V. 31, №. 89, p. 75-88, 2017.

ØSTERGAARD, C. R.; PARK, E. K. Cluster Decline and Resilience, - the case of the wireless communication cluster in North Jutland, Denmark

ÖZ, Ö.; ÖZKARACALAR, K. What accounts for the resilience and vulnerability of clusters? The case of Istanbul's film industry, European Planning Studies, V. 19, №. 3, p. 361-378, 2011.

PORTER, M. E. Clusters and the New Economics of Competition. Harvard Business Review. V. 76, № 6, pp. 77 - 90, 1998.

SAXENIAN, A. Regional Advantage: Culture and Competition in Silicon Valley and Route 128. Harvard Journal of Law \& Technology. V. 8, №. 2, p. 521 - 528, 1995.

SCHMITZ, H. Collective efficiency and increasing returns. Cambridge Journal of Economics. V. 23, pp 465 - 483, 1999.

SIMMIE, J.; MARTIN, R. The economic resilience of regions: towards an evolutionary approach. Cambridge Journal of Regions, Economy and Society, V. 3, p. 27-43, 2010.

STABER, U.; SAUTTER, B. Who Are We, and Do we Need to Change? Cluster Identity and Life Cycle. Regional Studies, V. 45, №. 10, p. 1349-1361, 2011.

SUIRE, R.; VICENTE, J. Cluster for life or life cycles of clusters: in search of the critical factors of cluster's resilience, Entrepreneurship \& Regional Development, V. 26, №. 1-2, p. 142-164, 2014.

Ter WAL, A. L. J.; BOSCHMA, R. A. Co-evolution of firms, industries and networks in space, Regional Studies, V. 45, №. 7, p. 919-933, 2011.

TÖDTLING, F.; TRIPPL, M. One size fits all? Towards a differentiated regional innovation policy approach, Research Policy, V. 34, p. 1203-1219, 2005.

TREADO, C. D. Pittsburgh's evolving steel legacy and the steel technology cluster, Cambridge Journal of Regions, Economy and Society, V. 3, p; 105-120, 2010.

TRIPPL, M.; OTTO, A. How to turn the fate of old industrial areas: a comparison of cluster-based renewal processes in Styria and the Saarland, Environment and Planning, V. 41, p. 1217 1233, 2009.

WOLFE, D. A. The strategic management of core cities: Path dependence and economic adjustment in resilient regions, Cambridge Journal of Regions, Economy and Society, V. 3, №. 1, p. 139-152, 2010.

WROBEL, M. One for all and all for one: Cluster, employment, and the global economic crisis. Evidence from the German mechanical enginnering industry, Papers in Regional Science, V. 94, №. 2, 2013.

YANG, C.; FU, T.; LI, L. Emerging Adaptation of Local Clusters in China in a Shifting Global Economy: Evidence from the Furniture Cluster in Houjie Town, Dongguan, Growth and Change, V. 48, №. 2, p. 214-232, 2017. 